\title{
Die Praktische Bedeutung ungarischer Sammlungen UND BibliotheKen FÜR FÜHRENDE GeLehrte DES KÖNIGREICHS UNGARN IM SPÄTEN 18. JAHRHUNDERT am Beispiel des Jesuiten Georg Pray (1723-1801)
}

\section{Andrea Seidler}

Für meine Analyse zur Bedeutung von Bibliotheken im wissenschaftlichen Diskurs im Königreich Ungarn im Zeitalter der Aufklärung habe ich vier Textsorten miteinander verflochten: gelehrte Korrespondenzen, Medien, handschriftliche Urkunden beziehungsweise Dokumente und die wissenschaftlíche Literatur des späten 18. bzw. frühen 19. Jahrhunderts. Meine Forschungsfrage lautet: Welche Bibliotheken oder öffentliche und Privatsammlungen standen den Gelehrten im Königreich Ungarn für ihre Forschungsarbeit zur Verfügung, welches Quellenmaterial war ihnen zugänglich, um ihre Arbeit voran zu treiben, in welchen Bereichen stießen sie auf Probleme oder gar auf Widerstand? Ich beziehe mich hier in erster Linie auf das gelehrte Netzwerk um den Pressburger Karl Gottlieb Windisch (1725-1793), der als Kommunalpolitiker und Geschäftsmann die in erster Linie private Ambition hatte, eine bedeutende, das Ungrische Magazin (1781-1787), ${ }^{1}$ - beziehungsweise davor bereits mehrere kleinere Zeitschriften herauszugeben und im Zusammenhang mit dieser Tätigkeit die berühmtesten Gelehrten des Königreichs Ungarn um sich zu versammeln. Der Protestant Windisch war auch der erste Redakteur der Preßburger Zeitung, 1764 erstmals herausgegeben. Alle diese Publikationen erschienen in deutscher Sprache.

Seine engsten wissenschaftlichen Mitarbeiter waren der Jesuit Georg Pray, weiters der Privatsekretär des Grafen József Teleki (17381796), Daniel Cornides (1732-1787), Historiker und Heraldiker, der

$1 \quad$ Karl Gottlieb Windisch (Hg.), Ungrische Magazin. Pressburg, Anton Löwe 17811787. Siehe dazu die online Plattform Hungarus Digitalis, https://www.univie.ac.at/ hungdigi/foswiki/bin/view.cgi/DigiHung/WebHome (gesehen am 2.3.2020) 
protestantische Siebenbürger Pfarrer Johann Seifert (1735-1785), Gottfried Kéler (1741-1807) und Johann Mathias Korabinszky (17401811), um nur einige zu nennen.

Georg Pray, Gelehrter und unglücklicher Bibliothekar

Georg Pray, dessen schriftlicher Nachlass, bestehend aus seinen Manuskripten sowie einer Vielzahl von Urkunden und Briefen, sich heute zum Großteil an der Universitätsbibliothek in Budapest befindet, möchte ich hier besonders hervorheben. ${ }^{2}$ Er ist der internationalen Forschung heute wenig bis gar nicht bekannt. ${ }^{3}$ Den eingeschränkten Publikationsmöglichkeiten für wissenschaftliche Arbeiten im Königreich Ungarn im 18. Jahrhunderts entsprechend, blieben die meisten Arbeiten Prays unveröffentlicht als Handschriften erhalten, wurden also auch zu seinen Lebzeiten nicht gedruckt. Vermutlich verdankte sich dies auch der lateinischen Sprache, an der er festhielt, und die zwar nicht unbedingt zeitgenössische, aber jedenfalls spätere Zugänge zu seinen wertvollen Arbeiten deutlich erschwerte. Selbst seine gedruckten Werke sind mittlerweile von der Forschung vergessen. (Siehe ein Verzeichnis seiner Werke im Anhang.)

2 Neben Georg Prays Nachlass an der Universitätsbibliothek von Budapest finden sich auch vereinzelte Briefe an der slowakischen Nationalbibliothek. Es kann davon ausgegangen werden, dass auch andere Sammlungen über Korrespondenzbelege Prays verfügen. Leider wurde die systematische Sammlung und Publizierung seines Briefwechsels noch nicht in Angriff genommen.

3 Siehe zu Prays Leben und Werk noch immer am umfassendsten Bibliographisches Lexikon des Kaiserthums Österreich, digital unter: https://de.wikisource.org/wiki/BLKÖ:Pray_Georg (Gesehen am 2. 3. 2020.) Ausführlich auch: Plutarch, Bildnisse und Bibliographien der berühmtesten Männer und Frauen aller Nationen und Stände; von dem älter bis auf unsere Zeiten, Pest 1844, S. 138ff. Jüngst erschien Paul SHore, Ex-Jesuit Librarian Scholars Adam Frantisk Kollár and György Pray: Baroque Tradition, National Identity and the Enlightenment among Jesuits in the Eastern Habsburg Lands, In: Journal of Jesuits Studies 6 (2019), S. 467-485. 


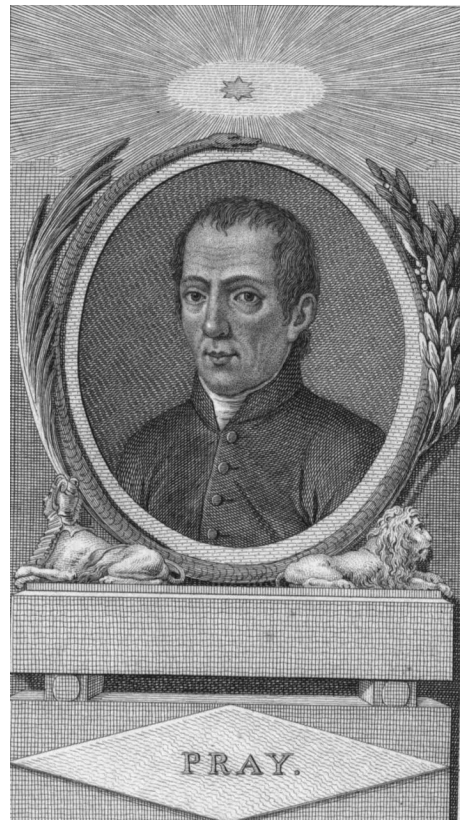

Die freundschaftliche Beziehung zwischen dem Protestanten Karl Gottlieb Windisch und dem Jesuiten Georg Pray dürfte lange vor dem ersten Quellenbeleg, einem Brief, den Pray 1778 an Windisch geschrieben hatte ${ }^{4}$ bestanden haben. Der vertraute Ton zwischen den beiden Männern, der zuweilen auch zynische Bemerkungen hinsichtlich politischer und religiöser Fragen zuließ, weist auf intensiven privaten Kontakt hin, hielt sich doch Pray, der an der Universität Tyrnau tätig war, auch häufig in Preßburg auf, war auch kurze Zeit dort beruflich engagiert. Pray war familiärer seits von adeliger Tiroler Herkunft und in Neuhäusel (Nové Zámky, Érsekújvár), im Königreich Ungarn geboren. ${ }^{5}$ Er trat am 14. Oktober 1740 in den Jesuitenorden, in das Collegium desselben zu St. Anna in Wien ein - damals siebzehnjährig. Später, in Tyrnau, wo er studierte, unternahm er erste Ansätze zur historischen Forschung: Vor allem das Sammeln, Kopieren und die Analyse von Dokumenten und Urkunden sowie die Altertumsforschung hatten es ihm angetan. In der Folge unterrichtete er am Wiener Theresianum, wo ihn Windisch, Budapest 2008 (Magyarországi tudósok levelezése - Briefwechsel ungarischer Gelehrter, 5) sowie die Internetplattform Hungarus Digitalis, https://www.univie.ac.at/hungdigi/foswiki/bin/view.cgi/DigiHung/ WebHome (gesehen am 2.3.2020)

5 József Antall, A Pray család magyarországi történetének összefoglaló ismertetése, In: Jászsági Évkönyv 13 (2005), S. 41-48. 
sein Lehrer Erasmus Fröhlich (1700-1758) ${ }^{6}$ eindringlich zum Studium der Erforschung der Geschichte drängte. Von da an beschäftigte sich Pray nur mit dem Beschaffen und Zusammenstellen des für die Verfassung seiner Werke nötigen Materials, wobei ihm auch durch das Machtwort der Kaiserin Maria Theresia die Benützung der Urkunden in allen Archiven Ungarns gestattet und ermöglicht wurde. Pray wurde in Tyrnau sogar von seinem Lehrkontingent befreit, um ungehindert forschen zu können. Seine Ausrichtung als Historiker war eine für die Habsburger durchaus vorteilhafte: Er war ein konservativer Vertreter seiner Forschungsrichtung, ein Befürworter alter - von Gott gegebener Werte, ein Unterstützer des Adels und deren Privilegien. Als Historiker, der sich in erster Linie mit der Geschichte der Ungarn und deren Ursprung beschäftigte, folgte er der hunnischen Herkunftstheorie der Ungarn - beeinflußt vor allem durch die Arbeiten des Franzosen Guignes (1721-1800), die bereits zu seinen Zeiten als eine Theorie, die eher auf Wahrscheinlichkeit als auf Fakten beruht, begewertet wurde.

Bald nach der 1773 erfolgten Aufhebung des Jesuitenordens erhöhte Maria Theresia Prays Pension von 200 auf 400 Gulden. und ernannte ihn zum Historiographen des Königreichs Ungarn (Historiographus Hungariae). ${ }^{8}$ Als im Jahre 1777 die Universität von Tyrnau nach Ofen und später nach Pest verlegt wurde, erhielt Pray den Ruf als erster Custos der Universitäts-Bibliothek dahin, dem er auch Folge leistete.

Erasmus Fröhlich, war ein aus Graz stammender Historiker, Bibliothekar und Numismatiker, der zahlreichen Studien absolviert hatte und ab 1746 an dem von Maria Theresia gegründeten Theresianum in Wien unterrichtete. Neben seiner Tätigkeit als Lehrer kümmerte er sich um die Ordnung der Bibliothek der Schule.

7 Joseph de Guignes, Histoire générale des Huns, des Turcs, des Mogols, et des autres Tartares occidentaux, Paris 1756.

8 Paul Shore (Anm. 2.) zu der Auflösung des Ordens und der damit einhergehenden Entwurzelung jesuitischer Gelehrter: „...by mid-century, Hungary and the Jesuit educational network within it had largely recovered. In this milieu the Jesuit educational network produced many important intellectuals, several of whom lived into the era following the Society's suppression.," S. 469 
Er war maßgeblich für die Übersiedlung, Auswahl und Systematisierung der Buchbestände zuständig - nicht alles wurde an einer nunmehr staatlichen Universität gebraucht, vieles zurückgelassen oder auch an diverse Diözesen verteilt. Der Umfang des übersiedelten Guts umfaßte immerhin ca. 10.000 Bände, war zeitgemäß ausgerichtet, da die Universität von Tyrnau ja auch in den Naturwissenschaften eine erhebliche Rolle gespielt hatte. Auch überließ Pray derselben seine eigene Sammlung von Büchern - über 2100 Stück - und Handschriften gegen eine jährliche Leibrente von 400 Gulden, um sie vor Zerstreuung und Verzettelung, die Schicksale so vieler Ordenssammlungen zu bewahren. Durch Pray gelangte auch die Sammlung des Gábor Hevenesi (Jesuitischer Gelehrter, 1656-1715) und István Kaprinai (ebenfalls Jesuit, 1714-1786) 1790 an die Universitätsbibliothek Ofen. Er stellte zusätzliche 60 Bände mit Urkunden zusammen, und durchforstete dafür das Archiv der Ungarischen Kammer in Preßburg. Außer der reichen Bibliothek und den Urkunden verfügte Pray über eine umfassende Brief- $-^{9}$ sowie eine Siegelsammlung. ${ }^{10}$ Diese Sammlung zählt heute zu den wichtigsten und wertvollsten Beständen der Universitätsbibliothek Budapest, hatten die drei Gelehrten doch versucht, einen möglichst umfassenden Bestand an historischen Belegen, die Geschichte des Königreichs Ungarn betreffend zusammen zu tragen, zu kopieren und zu systematisieren. Die Kollektion war 1768 aus Tyrnau in die Preßburger ungarische Kammer gelangt, auf Prays Initiative hin wurde sie in Ofen vereint. (Es handelte sich dabei um 15 Laufmeter, in erster Linie lateinisches Material.)

Die feierliche Eröffnung der Universität und der Bibliothek wurde am 25. Juni 1780 begangen. Die Pressburger Zeitung ${ }^{11}$ schrieb darüber in zwei Ausgaben ausführlich, Martin Schwartner (1759-1823) widmet ihr in seiner Statistik des Königreichs Ungern ebenfalls großen Raum:

$9 \quad$ Epistolae Procerum, 1806.

10 Syntagma historicum de sigilis, 1805.

11 Preßburger Zeitung, Preßburg, Landerer et al., ab 1764. In der Folge zitiert als PZ, hier 5. und 8. 7. 1780. 
„Seit dem [...] prächtigen Einzug des erwählten Königs Matthias v. Hunyad, sah Ofen kein glänzenderes Schauspiel innerhalb seiner alten Stadtmauern, als das gewesen ist, wozu die Einweihung der Universität in ihrem neuen königlichen Wohnorte, am 25 Junius 1780 die Veranlassung war. Gebenedeyet und gepriesen von Millionen Zungen, überlebte aber die große Kinder- und Schul Patroninn nur um 5 Monat die Freude, welche sie, beym Anblick der glücklich ausgeführten Erziehungsanstalten in ihrer großen Monarchie empfunden haben mag." ${ }^{\prime 2}$

Freilich war Maria Theresia bei dieser Feierlichkeiten nicht persönlich anwesend: Ein Gemälde, das ihr Porträt darstellte, stand an ihrer Stelle in Buda auf den samtenen Thron. Die Preßburger Zeitung schrieb dazu: "Allhier war ein majestätischer Thron mit einem rothseidenen und godbordierten Überzug aufgestellt, auf welchem das Bildniß der großen Theresia, von beiden Seiten mit Grenadieren bewacht, herrlich aufgerichtet war." ${ }^{\prime 3}$

Die Bibliothek wird in sämtlichen zeitgenössischen Medien aufs Höchste gelobt, Georg Pray selbst war allerdings von den Beständen seiner nunmehr neuen Wirkungsstätte nicht sehr begeistert.

Er schrieb darüber am 21. November 1778 an Karl Gottlieb Windisch nach Pressburg:

„Nun wo blieb ich vorhin? - - Bey der Bibliothek, die ich schildern wollte. Lauter Alterthümer, die zwar zur Zierde dienen, aber zum Gebrauche sind sie lauter o o o o, mit einem Worte, unsere Bibliothek sieht einem Weingarten gleich, wo Schauer, Hagel - - ohne die öden Plätze wo man noch manche Grubers anbringen könnte. Wo fehlt es denn? Etwa am Gelde? Es ist genug da, wann man es nur hergäbe - Jammer Schade, dass man die Besten und für das Vatterlande nützlichsten Absichten unserer weisesten Landesmutter so schläfrig der Diplomatik, und erstem Bibliotheks-Custos auf der Kön. ungrischen Universität zu Pest, Pest, gedruckt bey Matthias Trattner, 1789, §93. 
befolget: ich wünschte einen Hungarischen Colbert - dificile est non scribere, Sie wissen schon - und diese soll mit der Zeit eine öffentliche Bibliothek werden? Secula transibunt, transibunt secula donec est Decus et nomen Bibliotheca geret. Oder: ex merito."14

1781/82 verfasste Pray einen zwei Bänden umfassenden Katalog der besonderen Bestände der Universitätsbibliothek, der unter dem Titel Index rariorum librorum bibliothecae Univeraitatis Regiae Budensis in Ofen erschien. Die Allgemeine Deutsche Bibliothek setzte ihre Leserschaft durch eine längere Beschreibung des Verzeichnisses von dessen Existenz in Kenntnis. Die Nachricht beginnt mit einem Lob für den Verfasser, Pray: „Georgius Pray. Das ist der berühmte Exjesuit, dem die ungarische Geschichte so viel verdankt, und der der Vorsteher der königl. Bibliothek zu Ofen ist. [...] Der Hauptnutzen des Werkes erstreckt sich auf Ungarn und die Ofner Bibliothek, damit man weiß, welche raren Bücher darin sind und wer sie beschrieben hat. Denn außer den in Ungarn etablierten ist für den Ausländer wenig da," kritisiert der Verfasser.

Manche Bestände führte Pray an, obwohl die betreffenden Bände noch nicht in der Ofener Bibliothek eingelangt waren, sondern Maria Theresia ihm nur zugesichert hatte, aus den Beständen der aufgelösten Jesuitenbibliotheken Brauchbares auswählen zu könne. Der Rezensent wirft Pray, was diese zukünftigen Bestände angeht, Ungenauigkeit im Umgang mit den bibliographischen Angaben vor.

Aus dem Index geht die fachliche Zusammensetzung der Bibliothek hervor, wonach theologische und medizinische Bücher in der Überzahl waren, gefolgt von historischen und philologischen Beständen, „in dem letzten besonders finden sich manche Ausgaben alter Autoren von äußerster Seltenheit". Am dürftigsten sei es um das juridische Fach bestellt gewesen, schrieb die Allgemeine Deutsche Bibliothek. Prays im Brief an Windisch verklungene Kritik schlug sich also in seinem Index, der eine Sammlung vorstellte, die offenbar vor einem internationalen Leserpublikum nicht standhalten konnte, nieder. 
Pray war nicht nur wegen der Bestände unglücklich in Buda: Er wollte von seinem Posten als Custos entlastet werden und nur noch forschen. Windisch berichtet darüber an Daniel Cornides im Jahr 1781:

„Unser gemeinschaftlicher Freund der würdige P[ater] Pray, hat um seine Entlassung von der Universität angehalten, und solche auch, aber nicht das vorige Gehalt bekommen. Es bleiben ihm also nur seine alten 400 Gulden übrig, obgleich seine Freunde alles gethan haben, ihm eine größere Pension auszuwirken. Noch ist er unentschlossen, wo er seine künftige Wohnung aufschlagen wird, vielleicht in Siebenbürgen, wie er sich gegen mir geäußert hat.".

Und im Jahr 1782:

„Unser lieber Pray ist noch in Ofen; und man sucht ihn wieder da zu behalten. Einem Manne wie Pray ist, hätte man tausend Thaler jährlich ausweisen, und die Freyheit lassen sollen, nach seinem Belieben zu handeln. Aber gewiß keine Ehr für unser Vaterland! Doch vielleicht dringt Josephs scharfer Blick durch die Nebel der Schikane, und führt unsern Pray auf die Stelle, die für ihn bereitet war, aber durch andre besetzt ward. ${ }^{\prime 16}$

\section{Prays Reputation unter Zeitgenossen}

Kaiser Joseph II. belohnte Prays Abhandlung über Ungarns Ansprüche auf die an die Osmanen verlorenen Nebenländer mit einer jährlichen Zulage von 400 Gulden, aber in die gelehrte Autonomie ließ der Hof ihn nicht. Kaiser Leopold II. ernannte ihn im August 1790 zum Domherrn von Großwardein, in dieser Eigenschaft erschien Pray als Abgesandter seines Kapitels auf dem in demselben Jahre zu Pressburg abgehaltenen Reichstag.

Pray ist der historischen Forschung heutzutage weniger wegen seiner Verdienste als Geschichtsschreiber, als Historiograph, bekannt, sondern als Entdecker und erster Beschreiber von zwei der bedeutendsten Sprachdenkmäler der Ungarn, der Halotti Beszéd, (Totenrede, um 1192, 
erstes, vollständig auf ungarisch geschriebenes Sprachdenkmal, dzt. OSzK) sowie der Margit Legenda (Legende der Hl. Margit) auf die er im Zuge seiner Sammeltätigkeit gestoßen war ${ }^{17}$ - dem von der Nachwelt als Pray Kodex bezeichnetem Kompendium.

Pray war drüber hinaus wie erwähnt einer der wichtigsten Mitarbeiter des Ungrischen Magazins. Er wirkt in den Briefen an Karl Gottlieb Windisch als freizügig, humorvoll und setzt seine Spitzen und Pointen auch gegen die Obrigkeit privat nicht zu knapp.

Der Historiker Johann Christian Engel (1770-1814) gibt übrigens in seiner Geschichte des Ungrischen Reiches, Wien, 1803, umfassend Auskunft über die Sammlungen einzelner Gelehrter, sowie der öffentlichen Sammlungen im 18. Jahrhundert. ${ }^{18}$

In einem Vorwort zählt er Georg Pray zu den größten Geschichtsforschern des Königreiches, neben zahlreichen anderen wie Matthias Belius (1684-1749), János Tomka-Sászky (1692-1762), Sámuel Timon (1675-1736), Karl (Carolus) Wagner (1732-1790), Karl Gottlieb Windisch. Als Historiker beklagt er die Tatsache, dass zahlreiche bedeutende Werke zur ungarischen Geschichte nicht herausgegeben worden seien, dass es keine kritischen Ausgaben inländischer Chronisten gäbe, dass die Chronisten der benachbarten Länder in die kurrente Forschung nicht einbezogen würden und darüber, dass die Handschriften über die ungarische Geschichte nicht institutionell, beispielsweise durch eine Gesellschaft der Wissenschaften, gesammelt und gepflegt würden. Durchaus moderne Forderungen. Als die wertvollsten Sammlungen zählt er Matthias Belius Adparatus, von der ein Teil im Druck erschienen war. ${ }^{19}$ Die Originale wurden nach Belius' Tod verkauft, die meisten Urkunden und Dokumente an den Fürst Primas Erzbischof von Gran, József Batthyány (1727-1799), aber auch an private Sammler wie Ádam Franz Kollár (1718-1783).

19 Mathias Belius, Adparatus ad historiam Hungariae, Preßburg 1735. 
Rektor Joseph Benczur (1728-1784, Evangelisches Lyceum Preßburg), verfügte ebenfalls über eine ansehnliche Sammlung, die er vor allem seinen Studenten an der Evangelischen Schule in Preßburg verdankte, die die Originale kopierten. Sie ging später in die Sammlung des Freyherrn Alexander von Prónai (1760-1839) über. Samuel Bruckenthal (1721-1803), Daniel Cornides, Joseph Carl Eder (1761-1810), Gabriel Hevenesi, Stephan Kaprinai, Ádam Franz Kollár, Martin Georg Kovachich (1744-1821), Gottfried Kéler (1745-1807), Franz Graf Széchényi (1754-1820) besaßen ebenfalls Dokumente zur ungarischen Geschichte. Als einen der bedeutendsten Sammler stellte Johann Christian Engel allerdings Georg Pray vor.

\section{Die Kooperation zwischen Pray und Windisch}

Prays Zugang zu der Bibliothek und den Sammlungen waren natürlich auch für Windisch als Redakteur des Ungrischen Magazins und als Historiker von unschätzbarem Wert. Der oben erwähnte erste Brief von Pray an Windisch enthält zahlreiche Informationen zur historischen Kartographie sowie über die Geschichte und Geographie einiger ungarischer Komitate, kopiert aus alten Urkunden. Es ist anzunehmen, dass Windisch diese Angaben für seine Geographie des Königreichs Ungern benötigte. ${ }^{20}$ In einem Brief aus dem Jahr 1782 schickt Pray Berichtigungen zu Alexius Horányis (1736-1809) Beitrag über den Temescher Banat, der im Ungrischen Magazin, Band III/1 erschienen war. ${ }^{21}$ Er sandte Windisch eine Auflistung der Temescher (Temscher) und der Pressburger Grafen, die nach eigener Aussage ebenfalls seiner Sammlung von Urkunden entnommen worden waren. „Können Sie dieses Wirrwarr brauchen, so steht es Ihnen zu Diensten, nur müssen

20 Karl Gottlieb Windisch, Geographie des Königreichs Ungern, Preßburg 1780, 2 Tle.

21 Es ist unklar, um welchen Beitrag es sich handelt. Das vierte Stück des 2. Bandes enthält keine Arbeit über den Temescher Banat. Das erste Stück des 3. Bandes aber einen Beitrag Von den Temscher, und Pressburger Grafen. Ein Schreiben an den Herausgeber. Verfasser: unbekannt. Glaubt man Pray, so handelte es sich dabei um Alexius Horányi. 
Sie es verdeutlichen, und meinen Namen verschweigen"22, schrieb er an Windisch. Es handelt sich dabei um den nahezu wortgleichen Text des Beitrages Von den Temscher, und Preßburger Grafen. Schreiben an den Herausgeber. ${ }^{23}$

Pray war kein Freund von Alexius Horányis Arbeit und ihm gegenüber sehr kritisch eingestellt. ${ }^{24}$

Pray und Cornides ersuchten Windisch natürlich ebenfalls um Abschriften von Dokumenten, so beispielsweise aus der Sammlung des Erzbischöflichen Bibliothek zu Preßburg, in der der Nachlaß von Matthias Belius lag. Windisch konnte ihm allerdings nicht helfen. Er schrieb an Daniel Cornides über die Umstände in der Bibliothek:

„Gleich nach dem Empfange Ihres allerliebsten Briefes suchte ich unter dem Schutte von Papieren, den Eisenburger Comitat, in der so genannten Bibliothek des Kardinal Primas auf; aber leider! er war niergens zu finden; und sein Hauskaplan, der eine Art von Bibliothekar vorstellet (sein ehemaliger Bereiter) vermuthet, daß er solchen seinem Vetter geliehen habe. In dieser Bibliothek liegt alles, wie Heu und Streu untereinander, dort ein Buch, hier ein Pack Handschriften; einige Bücher stehen zerstreut in den Kästen, manche auf der Erde, und viele in Kisten! Horányi sollte ein Verzeichnis der Belischen M[anu]skr[ip]te machen, er fand aber kaum 2/3 davon. Schade, wann etwa einige davon verloren gegangen sind!"25

Die wertvolle Belius'sche Sammlung war demnach nahezu unbrauchbar geworden.

Pray versuchte Windisch 1778 auch zu überreden, in Preßburg selbst eine Bibliothek anzulegen. Er schrieb:

22 SeIdler, Briefwechsel, Windisch an Cornides, 7. 10. 1782.

23 Von den Temscher, und Preßburger Grafen, UM III/1, 1783. Verfasser: unbekannt (d.i. Pray), digital: https://www.univie.ac.at/hungdigi/foswiki/bin/view.cgi/ DigiHung/UngrischesMagazinBand3HeftlText02 (Gesehen am 2.3.2020)

24 Siehe dazu Szelester Nagy László, Horányi Elek levelezése, Budapest 2016. https://btk.ppke.hu/uploads/articles/911444/file/HoranyiLev.pdf (Gesehen am 2.3.2020)

25 Seidler, Briefwechsel, Windisch an Cornides, Anfang Februar 1783. 
„Zwey Köche verderben die Suppe! Das wissen Sie ja! Warum legen Sie keine Bibliothek in Preßburg an. Ich würde ihr meine Schriften, und Bücher widmen, es könnten sich mit der Zeit viele Betthüter finden, vielleicht würden ruhige Zeiten, und die beneficia corporis Christi et $S$. Andreae, würden dem publico mehr nützen als - Vale iterum." 26

Windisch selbst verfügte nachweislich über eine ansehnliche Privatbibliothek, deren Bestände Jozef Tancer anhand einer in Brünn befindlichen Inventarliste rekonstruierte. ${ }^{27}$ An die Gründung einer öffentlichen (im Sinne von offen für die Gelehrtenwelt) Bibliothek wagte er sich aber dennoch nicht heran.

Pray selbst starb im Jahr 1801, neun Jahre nach Windisch. Die Preßburger Zeitung widmete im zwei Zeilen: „Den 23. Sept. starb in Pest Herr Georg v. Pray, Abt der Hl. Jungfrau zu Tyrnau, Domherr von Großwardein und des Königreichs Ungarn berühmter Geschichtsschreiber, 73 Jahre alt."28

Liste der Werke Prays (Überarbeitete, dem BLKÖ übernommene Liste, siehe Fussnote 2)

De Institutione et Venatu Falconum Libri duo, Carmen didacticum (Tyrnaviae $\left.1749,8^{\circ}\right)$

Annales veteres Hunnorum, Avarum et Hungarorum ab Anno a. C. N. 210 ad Annum C. 997. deducti, Partes tres (Vindobonae 1761, Fol.)

Epistola responsoria ad dissertationem apologeticam Josephi Innocentii Desericii (Ince József Desericzky) auctoris Commentariorum de Initiis ac Majoribus Hungarorum (Tyrnaviae, 1762, $8^{\circ}$.)

Annales Regum Hungariae ab Anno Christi 997 usque ad Annum 1564 deducti, Partes V(Vindobonae, 1764-1770, Fol.);

Supplementum ad Annales veteres Hunnorum, Avarum et Hungarorum congestos (Tyrnaviae 1764, $8^{\circ}$.)

26 Seidler, Briefwechsel, Pray an Windisch, 21. November 1778.

27 Jozef TAnczer, Im Schatten Wiens, Zur deutschsprachigen Presse und Literatur im Pressburg des 18. Jahrhunderts, Bremen 2008.

28 PZ, 29.9.1801. 
Carmen eucharisticum, Benedicto Sajgho Archi-Abbati, vota solennia renovanti (Tyrnaviae 1767, Fol.)

Epistola responsoria ad partem Iam dissertationum Benedicti Cettonis e. S. J. (Tyrnaviae 1768, $8^{\circ}$.)

Dissertationes Desericii et Prayi de Origine Hungarorum, Tomi III (Colocae et Pestini 1768-1771, Fol.)

Vita S. Elisabethae viduae Landgraviae Thuringiae, nec non B. Margaritae Virginis; quarum illa Andreae II. haec Belae IV. Regis Hung. filia erat, Ex codicibus M. S. S. eruta ac praeviis dissertationibus illustrata (Tyrnaviae $1770,4^{\circ}$.)

Dissertatio, historico-critica de Sacra dextera D. Stephani I. Hungariae Regis, Adnexa vita S. Stephani auctore Chartuitio Episcopo, cum icone (Vindobonae 1771, $4^{\circ}$.)

Dissertatio historico-critica de Priorata Auranae, in qua origo, progressus, et interitus ex monumentis nondum editis explicantur (ab anno 1345 ad 1551) (Viennae $1773,4^{\circ}$.)

Dissertatio historico-critica de S. Ladislao Hungariae Rege (Posonii 1774, $4^{\circ}$.)

Dissertatio historico-critica de SS. Salamone Rege, et Emerico duce Hungariae (Posonii 1774, $4^{\circ}$.)

Dissertationes historico-criticae (decem) in Annales veteres Hunnorum, Avarum et Hungarorum (Vindobonae 1775, Fol.)

Supplementa ad Annales Hunnorum, Avarum et Hungarorum (Tyrnaviae 1775, Fol.)

Specimen Hierarchiae Hungariae, complectens Seriem chronologicam Archiepiscoporum et Episcoporum Hungariae, cum rudi dioecesium delineatione. Partes duae (I. de Archiepiscopatu Strigoniensi et ejus Suffraganeis. II. de Archiepiscopatu Colocensi et ejus Suffraganeis, Addita est Chorographia Patriarchatus Ipekiensis auctore Michaele Millisich (Posonii 1776 ad $1779,4^{\circ}$.)

Diatribe in dissertationem historico-criticam de S. Ladislao Hung. Rege Episcopatus Varadensis Fundatore ab Antonio Gánótzy conscriptam; Cui accedunt: Synodus sub Laurentio Strigon. et Episc. celebrata, et Conscriptio decimae Pontificiae in dioecesi Veszprim. (Posonii 1777, $4^{\circ}$.) 
Index rariorum Librorum Bibliothecae Universitatis Regiae Budensis. Partes duae cum Supplemento partis I. (Budae 1780-1781, 8.)

Imposturae CCXVIII. in dissertatione Benedicti Cetto de Sinensium Imposturis detectae et convulsae, Accedunt Epistolae anecdotae P. Augustini e Comitibus Hallerstein ex China scriptae (Budae 1781, $8^{\circ}$.)

Jacobo Mariosa (olim e. S. J.) Italiam repetenti Propemticon (Calocae 1781, 8.) ohne des Autors Wissen herausgegeben

Epistolae exegeticae Gregorii Pray, Stephani Katona et Danielis Cornides in dispunctionem Antonii Ganoczi cum Appendicula ad L. K. (Pestini 1784, 8.)

Taurica Juri Russico a Chatarina II. bello et pace asserta (Pestini 1787, $4^{\circ}$.) für diese Dichtung wurde Pray von der Kaiserin Katharina mit einer goldenen Schaumünze beschenkt

Taurunum auspiciis Josephi II. recuperatum, Poemation, cum icone urbis (Pestini 1789, $4^{\circ}$. maj. Fol.)

Historia Controversiarum de ritibus Sinicis, ac earum origine ad finem compendio deducta, praecedente epistola ad Benedictum Cetto (Pestini 1789, 8.) - auch unter dem Titel: Epistola ad Benedictum Cetto, in qua novae hujus in rebus Sinicis Imposturae deteguntur. Accedit Historia Controversiarum etc. (ibid. 1789, $8^{\circ}$.) - deutsch in 3 Bänden (Augsburg 1791, $8^{\circ}$.)

Reflexiones super declaratione sincera christiana et patriotica Civis Hungari Catolici ad questionem: An sic dicta apostasia inter delicta civilia referenda occasione Gen. Reg. Commitiorum (sine loco, 1790, 8.), ohne Angabe des Autors und Druckortes, die neue Fachliteratur vermutet den Benediktiner Tóbiás Mollik (1751-1824) als Verfasser:

http://nektar.oszk.hu/hu/manifestation/3232093

Ad Amicum Augustanae Confessionis amici catolici de Viennensi et Linciensi Pacificatione, Epistolae III (1790, 8.) - ohne Angabe des Autors und Druckortes, aber die neue Fachliteratur vermutet den Jesuiten István Katona (1732-1811) als Verfasser des Werkes: Posonii, Simon Peter Weber, 1791 (http://nektar.oszk.hu/hu/manifestation/3128788). Die zeitgenössische Quelle schreibt es Pray zu, siehe Syntagma Historicum de Sigillis Regum, et Reginarum Hungariae pluribusque aliis autore Georgio Pray, Budae 1805. 
Animadversiones in libellum (Danielis Crudy) cui titulus: Sola salvifica ad trutinam rationis et revelationis expensa, ad rectum revelationis et rationis usum exacta (sine loco, 1790, $8^{\circ}$.)

Ad Auctorem nullitatis animadversionum (Danielem Crudy Posoniensem Superintendentem) in libellum cui titulus: Sola salvifica ad trutinam rationis et revelationis expensa ... epistolae tres familiares (Pestini 1791, $8^{\circ}$.)

Historia Regum Hungariae cum notitiis praeviis ad cognoscendum veterem Regni Statum pertinentibus etc. in partes tres divisa (Budae 1801, $8^{\circ}$.)

Horszuvaryi (Georgii Pray) Responsio ad Recensionem Novorum Univ. Litter. Jenaensium super libro Georgii Pray Historia Regum Hungariae stirpis Austriacae (Viennae 1801, 8.) In deutscher Sprache.

Nach seinem Tode wurde herausgegeben:

Syntagma historicum de Sigillis Regum et Reginarum Hungariae, pluribusque aliis ... cum XVI. Tabulis aeri incisis, Accedunt 1) Vitae ac scriptorum auctoris recensio (per Michaelem Antonium Paintner), 2) Series chronologica Cancellariorum et Vice-Cancellariorum Hungariae a condito Regno ad hanc aetatem perducta, 3) Vetera duo calendaria in usum Ecclesiae Strigoniensis (Budae 1805, $4^{\circ}$.)

Epistolae Procerum Regni Hungariae ab anno 1490 ad annum 1771 collectae, Tomi III (Viennae et Pestini 1805 et 1806, $8^{\circ}$.) 
LES BIBLIOTHÈQUES ET L'ÉCONOMIE DES CONNAISSANCES Bibliotheken und die ÖKonomie des Wissens

$$
145^{\circ}-185^{\circ}
$$

Colloque international - Internationale Tagung 9-13 avril/April 2019 Sárospatak (Hongrie/Ungarn)

Édité par

Frédéric Barbier, István Monok et Andrea Seidler 


\section{L'Europe en réseaux}

Contribution à l'histoire de la culture écrite 1650-1918

Vernetztes Europa

Beiträge zur Kulturgeschichte des Buchwesens 1650-1918

Édité par

Frédéric Barbier, Marie-Elisabeth Ducreux, Matthias Middell, István Monok, Éva Ringh, Martin Svatoš

Volume VIII

École pratique des hautes études, Paris

École des hautes études en sciences sociales, Paris

Centre des hautes études, Leipzig

Bibliothèque nationale Széchényi, Budapest

Bibliothèque et centre d'information de l'Académie hongroise des sciences, Budapest 
LeS BIBLIOTHÈQUeS ET L'ÉCONOMIE DES CONNAISSANCES BibliotheKen und die ÖKonomie des Wissens

$$
145^{\circ}-185^{\circ}
$$

Colloque international - Internationale Tagung 9-13 avril/April 2019 Sárospatak (Hongrie/Ungarn)

Édité par

Frédéric Barbier, István Monok et Andrea Seidler

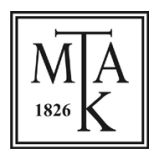

Magyar Tudományos Akadémia Könyvtár és Információs Központ Budapest 2020 
Mise en page

Viktória Vas

ISBN 978-963-7451-57-7

DOI 10.36820/SAROSPATAK.2020 
Préface

István MoNOK

Bibliothecae mutantur - Quare, quemadmodum et quid attinet?

Transformations de la composition thématique

des bibliothèques du Royaume de Hongrie aux $\mathrm{XV}^{\mathrm{E}}-\mathrm{XVI} \mathrm{I}^{\mathrm{e}}$ siècles....11

Marianne CARbonnier-Burkard

Les bibliothèques des Églises réformées françaises au XVII siècle.... 30

Max Engammare

De la bibliothèque de l'Académie de Calvin (1570) a la bibliothèque de l'Académie de Bèze (1612) à travers leur catalogue: Continuités et ruptures jusqu'au troisième catalogue de 1620........... 57

Róbert OLÁ́

Obsolescent Reformed Libraries in the seventeenth and eighteenth Century Carpathian Basin

Ádám Hegri

Moderner Zeitgeist - veraltete Lesestoffe. Bibliotheken reformierten Pfarrer um die Wende des 18. zum

19. Jahrhunderts im Königreich Ungarn

Petr MAšEK

Zierotin Library in Velké Losiny in Sixteenth and Seventeenth century. 136

Detlef HABERLAND

Schlesische Bibliotheken Zeichen der intellektuellen Vielfalt einer zentralen Bildungsregion in Europa 146

Thomas WaLLNIG

Sebastian Tengnagel und Johann Seyfried - Österreichische

Geschichtsschreibung zwischen Späthumanismus und

Gegenreformation 
Elisabeth EngL-Ursula RautenBerg

Christoph Jacob Trew - Bibliothek und Sammeln in der Gelehrtengemeinschaft der ersten Hälfte des 18. Jahrhunderts.

Helwi BLOM

Philosophie ou Commerce?

L'évolution des systèmes de classement bibliographique dans les catalogues de bibliothèques privées publies en France au XVIII ${ }^{\mathrm{e}}$ siècle.

Maria Luisa López-VIDrIero Abelló

Les meubles de la connaissance: façons de devenir sage à prix fixe.

Frédéric BARbier

Distinction, récréation, identité: la trajectoire des « romans» en France sous d'Ancien Régime.

\section{Andrea SeIdler}

Die praktische Bedeutung ungarischer Sammlungen und Bibliotheken für führende Gelehrte des Königreichs Ungarn im späten 18. Jahrhundert am Beispiel des Jesuiten Georg Pray (1723-1801).

\section{Olga Granasztói}

Se divertir: les enseignements de la bibliothèque d'une femme aristocrate hongroise à la fin du XVIII siècle

Christophe Didier

La naissance du théâtre „des boulevards”, ou Comment la banlieue entre en bibliothèque (1780-1830).

\section{Andrea De Pasquale}

La nascita delle riserve di libri antichi in Italia

Index des noms de personne et de lieu..... 